\title{
The rp-process in Type-I X-ray Bursts with REACLIB V1.0
}

\author{
Zachary P. Meisel $^{\star a b c}$, Richard H. Cyburt ${ }^{a b}$, R. Ferguson ${ }^{a b c}$, S. Warren ${ }^{a b d}$, A.M. \\ Amthor $^{a b c}$, K. Smith ${ }^{a b c}$, J. Fisker ${ }^{e f}$, A. Heger ${ }^{a g}$, E. Johnson ${ }^{c}$, T. Rauscher ${ }^{h}$, A. \\ Sakharuk $^{a b i}$, H. Schatz ${ }^{a b c}$, F.-K. Thielemann ${ }^{h}$, and M. Wiescher ${ }^{a f}$ \\ ${ }^{a}$ The Joint Institute for Nuclear Astrophysics(JINA) \\ ${ }^{b}$ The National Superconducting Cyclotron Laboratory (NSCL) \\ ${ }^{c}$ Department of Physics and Astronomy, Michigan State University \\ ${ }^{d}$ Department of Electrical and Computer Engineering, Michigan State University \\ ${ }^{e}$ Lawrence Livermore National Laboratory \\ ${ }^{f}$ Department of Physics, University of Notre Dame \\ ${ }^{g}$ Theoretical Physics Group, Los Alamos National Laboratory \\ ${ }^{h}$ Department für Physik und Astronomie, Universität Basel \\ ${ }^{i}$ Department of Physics, Florida Gulf Coast University \\ E-mail: meisel@nscl.msu.edu, cyburtenscl.msu.edu
}

\begin{abstract}
Nuclear astrophysics is a rich and diverse field of research, requiring knowledge of experiments, observations, and modeling. Of key importance to modeling is the nuclear physics input, whether it be thermonuclear reaction rates or weak decay rates. The Joint Institute for Nuclear Astrophysics (JINA) has taken a leading role in maintaining an up-to-date database of these rates. The database can be accessed at the location: http://www.nscl.msu.edu/ nero/db/. The JINA REACLIB Database, based on F. Thielemann's 1995 REACLIB, has been updated to incorporate the latest weak decay data, as well as new experimental/theoretical reaction rates relevant for various nucleosynthetic processes. Included in the newly released REACLIB V1.0 library are several rates which have been fit using a new method which pays close consideration to physicality. In order to assess the impact of the new rates, comparisons were made against an older reaction rate library via X-ray burst simulations. Being heavily dependent on thermonuclear reaction rates, $\mathrm{X}$-ray burst simulations are ideal for such a comparison.
\end{abstract}

10th Symposium on Nuclei in the Cosmos

July 27 - August 12008

Mackinac Island, Michigan, USA

\footnotetext{
* Speaker.
} 
X-ray bursts are thermonuclear explosions which frequently occur on neutron stars in accreting binary systems [14]. Astronomers readily observe these phenomena due to their high luminosity ( $\sim 10^{38} \mathrm{erg} / \mathrm{s}$ ) and short recurrence time (hours to days). The nucleosynthesis occurring at these sites is governed by the rp-process, which involves many reaction rates recently updated in the JINA REACLIB database [1] in Version 1.0.

The primary thermonuclear reactions within the rp-process are the $(\mathrm{p}, \gamma)$ and $(\alpha, \mathrm{p})$ reactions, which collectively work to push element formation along the neutron deficient side of the valley of stability, and $\beta^{+}$decays, which push the element formation back toward the valley. New data on $(\mathrm{p}, \gamma)$ and $(\alpha, \mathrm{p})$ reactions from various sources [2], such as Iliadis et al. [3], were used to update hundreds of reaction rates in the REACLIB database, such as those previously provided by Caughlan and Fowler [4] and the NACRE [5] collaboration. New weak decay information from the 2007 Nuclear Wallet cards [6] was incorporated to provide the most up to date $\beta$ decay rates. New masses were also used, taking into account new experimental results and theoretical predictions [7], along with an updated NON-SMOKER model [8] to produce new rates. Given the relevance of these changes to the rp-process, we decided it was prudent to record the effect of these changes on simulated $\mathrm{x}$-ray burst light curves.

Rates involved in this study were represented in the seven-parameter REACLIB format:

$$
N_{A}<\sigma v>=\exp \left[a_{0}+\sum_{i=1}^{5} a_{i} T_{9}^{\frac{2 i-5}{3}}+a_{6} \ln T_{9}\right]
$$

Note that multiple exponential sets can be summed for a single reaction rate, with each exponential set representing a non-resonant or resonant contribution.

This format is capable of handling all reaction types. Non-physical behavior is avoided by enforcing physical constraints on the fit parameters. The most basic of these constraints provides separate sets of fit parameters for each non-resonant and narrow resonant contribution. It is possible to combine multiple narrow resonances in a single set for simplicity in presentation by first using the fit parameters of the highest energy resonance and then allowing these values to float. Similarly, in the combination of non-resonant sets, parameters of the most dominant set are used in the initial fit and are then allowed to float. Regarding the fit parameters themselves, $a_{0}$ through $a_{6}$, the rules vary depending on whether or not the set is charge-induced or neutron-induced and whether the set is resonant or non-resonant. The following are the general guidelines for fitting +Q-value reactions:

- Charge-Induced Non-Resonant:

- $a_{1}=0, a_{6}=-2 / 3$, and $a_{3}, a_{4}, a_{5}=$ float

- $a_{0}=\ln \left[7.8318 \times 10^{9}\left(Z_{1} Z_{2} / A\right)^{1 / 3} S_{0}\right]$

$-a_{2}=-4.2486\left(Z_{1}^{2} Z_{2}^{2} \mathrm{~A} / T_{9}\right)^{1 / 3}$

- Neutron-Induced Non-Resonant:

- $a_{1}, a_{2}=0$, and $a_{3}, a_{4}, a_{5}=$ float

- $a_{0}=\ln \left[N_{A} C^{\ell} \frac{\Gamma(\ell+3 / 2)}{\Gamma(3 / 2)}\left(\frac{\sigma v}{E^{\ell}}\right)_{E=0}\right]$

- $a_{6}=\ell$, the lowest possible orbital angular momentum 
- Narrow Resonant:

$$
\begin{aligned}
& -a_{6}=-1.5, \text { and } a_{2}, a_{3}, a_{4}, a_{5}=0 \\
& -a_{0}=\ln \left[1.5394 \times 10^{11} A^{-3 / 2} \omega \gamma\right] \\
& -a_{1}=-11.6045 E_{r}
\end{aligned}
$$

In the previous equations $N_{A}$ is Avogadro's number, $\sigma$ is the cross section in $\mathrm{cm}^{2}, \mathrm{v}$ is the center of mass relative speed in $\mathrm{cm} / \mathrm{s}, \mathrm{E}$ is the center of mass relative energy in $\mathrm{MeV}, Z_{1}$ and $Z_{2}$ are the reactant charges, $\mathrm{A}$ is the reduced mass of the reactants in atomic mass units, $S_{0}$ is the astrophysical S-factor at zero energy, $\omega \gamma$ is the narrow resonance strength in $\mathrm{MeV}, E_{r}$ is the narrow resonance energy in $\mathrm{MeV}$, and $\mathrm{C}=0.08617 \mathrm{MeV}$. A more comprehensive description of parameter selection and rate fitting procedure can be found in [8].

As an example we present the reaction rate for ${ }^{32} \mathrm{Cl}(\mathrm{p}, \gamma)$ and briefly discuss its four versions in the REACLIB database. This rate has two experiment-based versions and two theory-based versions. The labels sb05, hg95, rath, and ths 8 correspond to the references $[9,10,11,12]$, respectively. Regarding the experimental rates, sb05 and hg95, sb05 has been updated to include more recent resonance energies and strengths. Also new with the sb05 rate is the inclusion of excited states and a stellar enhancement factor (SEF), thus making it the recommended rate. As an aside, it should be noted that recommended rates are chosen by comparing the merits of each rate and recommending the one which seems to incorporate the most recent, reliable data and calculations. Regarding the theoretical rates, rath and ths8, ths8 uses an improved description of spins and excited states, an updated Q-value, and a new NON-SMOKER model [8]. The versions are presented for comparison in the following graph of $\log _{10}$ (Reaction Rate) vs. Temperature, noting that REACLIB fits are within 5\% of their respective data. Also noteworthy are the visible separations in the fits, which correspond to large differences in the reaction rate and, consequently, illustrate the impact of the update.

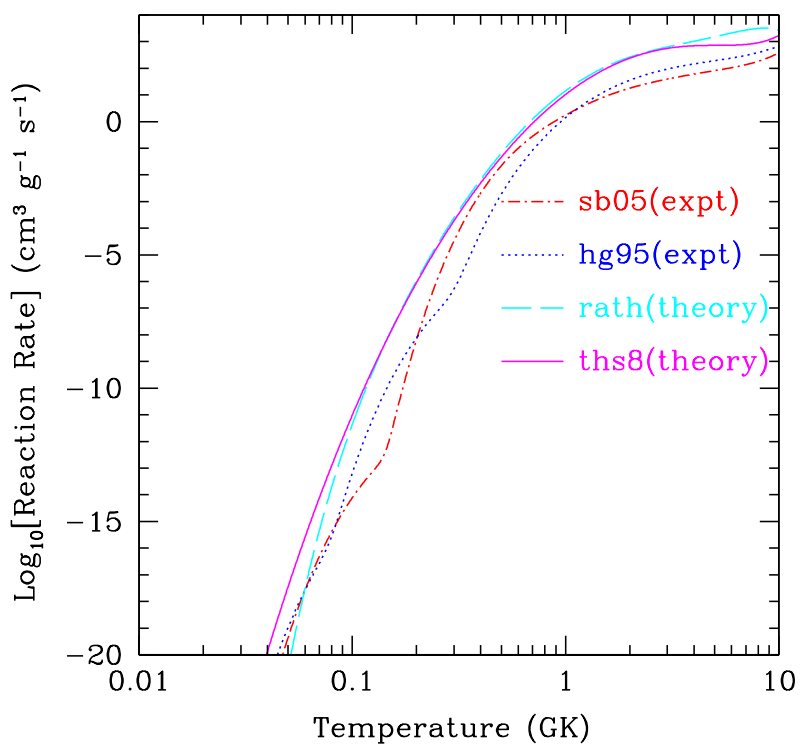

FIGURE 1: Four versions of the ${ }^{32} \mathrm{Cl}(\mathrm{p}, \gamma)$ reaction. 
In the recent REACLIB update to V1.0, thousands of rates spanning the nuclide chart were altered; many of which have potential impacts on the rp-process. Details of the V1.0 contents can be viewed at the JINA REACLIB database website [1]. To explore the impact of the update on x-ray burst models, we compare light curves simulated by computer models containing thermonuclear reaction rates from various REACLIB libraries. Single-zone [13] simulations were run for the REACLIB200, REACLIBV0.5, and REACLIBV1.0 libraries. Multi-zone [15] simulations were run for the REACLIBV0.5 and REACLIBV1.0 libraries. The result is shown in Figure 2. One should note that bursts were shifted to peak at the same time and the downward spike in the Multizone REACLIBV0.5 tail is a numerical artifact.

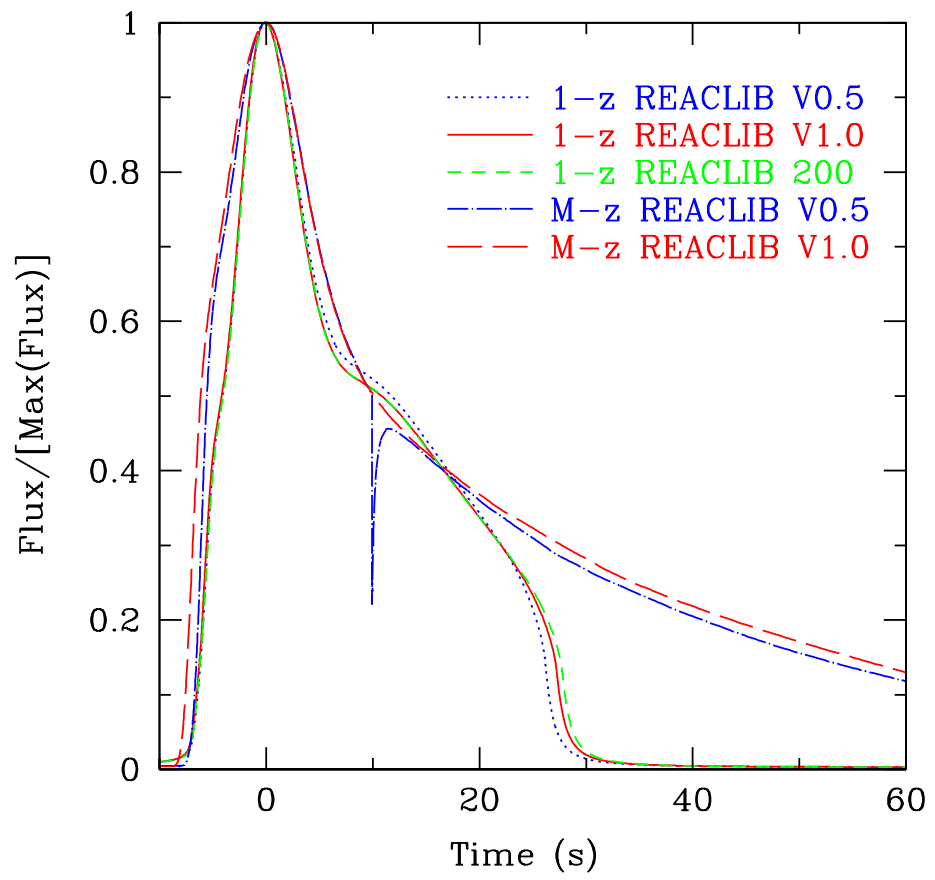

FIGURE 2: Simulated x-ray burst light curves.

It is apparent that the greatest observable changes for the multi-zone simulations occur in the rise of the light curves, with the V1.0 simulation rising more quickly. This suggests that the biggest impact comes from rate changes involving nuclei below ${ }^{56} \mathrm{Ni}$ on the nuclide chart. For the single-zone simulation, the tails of the light curves contain the largest changes. This suggests that reactions involving nuclei heavier than ${ }^{56} \mathrm{Ni}$ provide the greatest impact on the single-zone simulations. Peak luminosity is also affected by the library change, however, normalization obscures this. Quantitatively, the multi-zone V1.0 peak is $1.2 \%$ lower than the V0.5 peak and the single-zone V1.0 peak is $1.3 \%$ and $0.2 \%$ lower than the V0.5 and V200 peaks, respectively.

Future use of the REACLIB database is greatly encouraged due to its multiple advantages. Most importantly, the database is frequently updated with information from new research, ensuring that the reaction rates being used are the most up to date as possible. The database is highly accessible due to its public availability and numerous download formats, such as BDAT, XML, REACLIB1, and REACLIB2. The database can be accessed at http://www.nscl.msu.edu/ nero/db. 
The improved fitting method and new input data included in the V1.0 library in JINA's REACLIB database provide an important addition to the nuclear astrophysics community and should serve as a useful tool for modelers. Future prospects for the database include extending the fitting range by increasing the number of parameters to nine, adding new rates relevant to $r$ and s-process nucleosynthesis, and including reaction rate uncertainties. These possibilities increase the potential impact JINA's REACLIB database could have on the nuclear astrophysics community.

\section{Acknowledgments}

We would like to thank the following for their contribution to this work through informative conversations: Michael Smith and the participants in the X-Ray Burst poster room for NICX. This project is funded by the National Science Foundation through grants PHY0216783(JINA) and PHY0110253(NSCL).

\section{References}

[1] http://www.nscl.msu.edu/ nero/db/

[2] http://www.nscl.msu.edu/ nero/db/viewList.php?listID=12

[3] C. Iliadis et al. Astrophysical Journal 134 (2001), 151

[4] G. Caughlan \& W. Fowler ADNDT 40 (1988), 283

[5] C. Angulo et al. Nuclear Physics A A656 No.1, 3

[6] J. Tuli NNDC 2007 Nuclear Wallet Cards http://www.nndc.bnl.gov/wallet/

[7] B. Brown et al. Physical Review C 65 (2002), 045802

[8] R. Cyburt et al. To be published.

[9] H. Schatz et al. Physical Review C 72 (2005), 065804

[10] H. Herndl et al. Physical Review C 52 (1995), 1078

[11] T. Rauscher \& F.-K. Thielemann ADNDT 75 (2000), 1

[12] T. Rauscher Private communication.

[13] H. Schatz et al Physical Review Letters 86 (2001),1

[14] T. Strohmayer \& L. Bildsten New Views of Thermonuclear Burstseds. W. Lewin \& M. van der Klis (Cambridge University Press, Cambridge, MA. 2006) pp.113

[15] S. Woosley et al. Astrophysics Journal Supplement Series 151 (2004), 75 